DOI 10.1007/s00167-005-0029-y

\author{
Raffaele Garofalo \\ Elyazid Mouhsine \\ Pierre Chambat \\ Olivier Siegrist
}

\section{Anatomic anterior cruciate ligament reconstruction: the two-incision technique}

Received: 18 April 2005

Accepted: 28 June 2005

Published online: 4 January 2006

(C) Springer-Verlag 2006

R. Garofalo · O. Siegrist

Hopital Orthopedique de la Suisse

Romande, Avenue Pierre Decker 4, 1005 Lausanne, Switzerland

P. Chambat

Clinique Saint'Anne Lumiere, Lyon,

France

E. Mouhsine $(\square)$

Centre Hospitalier Universitaire Vaudois,

OTR-BH 14, CHUV,

1011 Lausanne, Switzerland

E-mail: elyazid.mouhsine@hospvd.ch

\begin{abstract}
This article describes the anatomical two-incision reconstruction of anterior cruciate ligament (ACL) of knee. The major part of currently single incision tibial endoscopic techniques attempts to reproduce the most isometric anteromedial bundle of ACL. Often a relatively vertical femoral tunnel, respect to the notch, is drilled, which is not really efficacious in providing rotatory stability. The single incision technique was developed to obviate the necessity of the lateral femoral incision and dissection. This technical note describes a two-incision ACL reconstruction using an instrumentation, which avoids a
\end{abstract}

large lateral femoral soft tissue dissection, and discusses the rational use of the two-incision ACL reconstruction technique.

Keywords Anterior cruciate ligament reconstruction - Anatomic reconstruction $\cdot$ Two-incision technique

\section{Introduction}

Many techniques of reconstruction of the anterior cruciate ligament (ACL) have been developed. Traditionally, arthroscopically assisted ACL reconstruction has been performed using a two-incision technique $[4,6]$. More recently, however, the single incision tibial endoscopic technique has been developed to obviate the necessity of the lateral incision and to, potentially, reduce operative time and surgical morbidity.

Nevertheless, endoscopic methods have shown some disadvantages with respect to the two-incision techniques. These include the inability to freely position the femoral tunnel when it is drilled through the tibial tunnel. Moreover, this technique dictates relatively vertical and central non-anatomical graft placement compared to the more horizontal and lateral course of the native ACL that could result in a rotational instability $[22,25]$.
Some authors recommend fluoroscopic control during surgery $[5,19]$ to avoid placing a femoral tunnel too shallow along the Blumensaat line. The use of anteromedial portal instead of transtibial portal has been suggested to obviate the above-mentioned problems. Through this portal with the knee maximally flexed, seems to be possible to perform a femoral tunnel $5 \mathrm{~mm}$ anterior to the posterior capsular insertion, at the 11 o'clock (right) or 1 o'clock position with respect to the apex of the notch [28].

Nevertheless there are other recognized potential pitfalls of the endoscopic technique, including graft tunnel mismatch, [10] interference screw fixation divergence, [1] posterior cruciate ligament (PCL) impingement [7] and possible violation of posterior cortical wall [26].

In this paper we present our technique for anatomic reconstruction of the ACL using the two-incision approach. In particular, a specific instrumentation to 
perform the femoral tunnel is used to avoid a large lateral femoral soft tissue dissection.

\section{Surgical technique}

\section{Surgical setup}

The patient is placed in the supine position with a lateral post just proximal to the knee, in level with a padded tourniquet and a foot roll helping to keep the hip from externally rotating and the knee flexion at $90^{\circ}$. In this way, the knee can be moved freely right through its full range of motion. Antibiotic prophylaxis is prepared with $1.5 \mathrm{~g}$ of a second generation cephalosporin or $1 \mathrm{~g}$ vancomycin for the patient who is allergic to penicillin. A thorough examination of both knees is performed under anaesthesia, including Lachmann, varus/valgus stress and pivot shift test to further confirm the diagnosis. Standard arthroscopic draping is used.

\section{Graft harvesting}

We start the surgery with a graft harvest (usually bonepatella tendon-bone (BPTB)). An incision of average $7 \mathrm{~cm}$ is performed, extending from the inferior pole of patella to the tibial tubercle, keeping the knee in flexion. The paratenon is identified and divided longitudinally and dissected medially and laterally only till the $10 \mathrm{~mm}$ width that allows graft harvest. A BPTB graft is harvested $10 \mathrm{~mm}$ in diameter with 10 or $11-\mathrm{mm} \times 25-\mathrm{mm}$ bone plug at the level of tibial tuberosity and 9 or 10$\mathrm{mm} \times 15-\mathrm{mm}$ bone plug at the level of patella. A single scalpel is used to outline the bone block that has to be cut on the tibial tubercle. The patella and bone block are harvested by using an oscillating saw. The block is cut in a trapezoidal fashion at the level of tibial tubercle and in a triangular fashion at the level of patella. After completion of the graft harvest, the patellar tendon defect and paratendon is closed with 0-vicryl in a running fashion. Closing the tendon and the paratendon at this stage helps prevent leakage of fluid and maintains distension of the joint during the arthroscopy. At the rear table, an assistant prepares the graft. A small rongeur and a graft shaper are used to fit the appropriately sized hole of a sizing block. It is very important to maximize the size of the bone plugs within the respective tunnels to obtain rigid fixation of the graft. The longer end tibial tuberosity of the graft is prepared as press-fit that goes into the femoral tunnel. In particular, the half part of bone plug nearest to the tendon is of the same diameter as that of the femoral tunnel and is shaped so as to easily pass in the femoral tunnel, whereas the other half part is $1 \mathrm{~mm}$ thicker than femoral tunnel diameter to allow press fitting of the bone block. A drill hole, about $5 \mathrm{~mm}$ from the end of bone block, is made with a 2-mm drill bit, and a holding suture (2-vicryl) is passed through it. The patella bone block is prepared not in press-fit; but two drill holes, evenly spaced that are made through the bone block parallel. Normally patellar bone plug is 1 or $2 \mathrm{~mm}$ smaller than tibial bone plug. A 2-vicryl suture is passed in an eight shape and a knot is performed at the extremity of bone block. This configuration allows this bone block to be pulled during graft passage. The tendoosseous junction at the level of tibial tuberosity plug that will be inserted in press-fitting into the femoral tunnel is marked with a sterile marker pen (Fig. 1).

\section{Arthroscopic reconstruction}

Inferomedial and inferolateral portal are made through the incision performed for graft harvesting eliminating the need for a separate portal incisions. A suction cannula is inserted through the suprapatellar pouch to end in the medial gutter. Sufficient debridement of the fat pad is performed to allow a good view of the intercondylar notch. Any remaining fibres of the ruptured ACL are then debrided using arthroscopic scissors and motorized $4.5 \mathrm{~mm}$ full radius resector. The medial wall of lateral condyle is debrided of all tissue. A rugine is used to well clear the lateral wall to help visualize the posterior notch. Care is taken through the debridement to avoid damage to the PCL and meniscofemoral ligaments. Although we work under tourniquet, sometimes a radiofrequency ablation device is used to clear tissue and provide haemostasis. During notch preparation, a probe is used to hook over the top. Once good arthroscopic view of the more posterior aspect of femoral lateral condyle is obtained, the tunnels are created at the origin of the ACL, starting from the femoral tunnel.

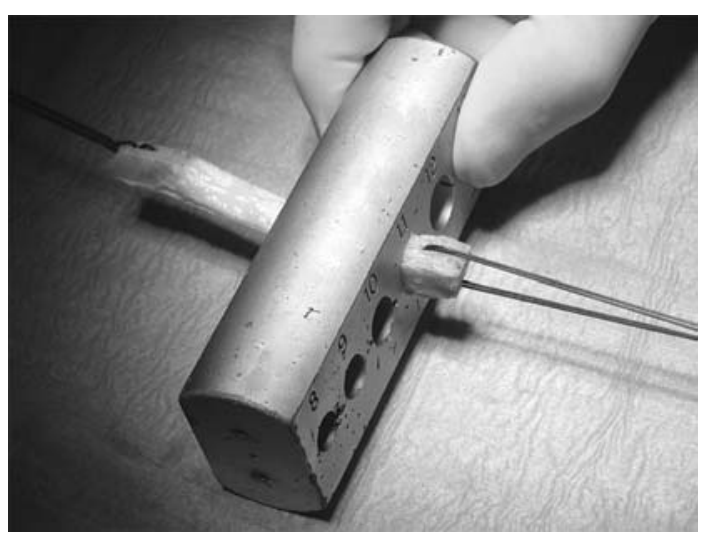

Fig. 1 Whether the distal parts do not pass, showing a good preparation for press-fit fixation 
Femoral pin guide

A specific femoral drill guide (Phusis, Grenoble, France) is positioned under arthroscopic control, through the anteromedial portal. One arm of the guide is introduced into the knee, passing between the LCP and the medial wall of lateral condyle. The tip of arm is positioned at the back wall of the lateral condyle. The knee is flexed at $90^{\circ}$. The tip of the guide is hooked just a few millimetres lower from the top position, immediately above the edge of lateral femoral condyle cartilage between 9 and 10 o'clock position for right knee and 14, 15 o'clock position for left knee, respectively. The external arm of the femoral guide lies on the lateral aspect of the lower part of the thigh. A lateral skin incision of average $2 \mathrm{~cm}$ is made along the lateral aspect of the distal femur; the point is dictated by the femoral guide (Fig. 2). The incision is straight to the bone; the iliotibial band is split longitudinally. The boundaries inferior and posterior of the lateral femur incision are represented by the higher insertion of the lateral collateral ligaments and posterolateral complex thus determining a tunnel, starting at the level of distal metaphysis of the femur. The guide pin is drilled with a slight oblique direction from front to back and from high to low. Pin position is confirmed arthroscopically by probing and direct visualization and the drill guide is disengaged.

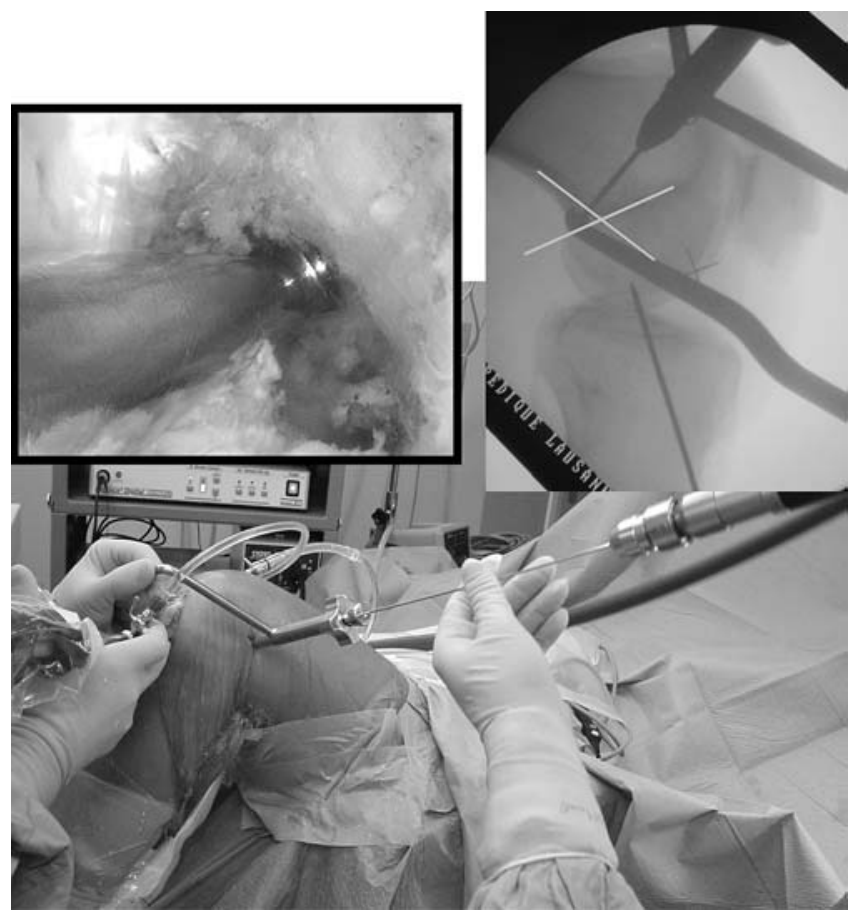

Fig. 2 Femoral guide wire is drilled from a lateral incision. Left arthroscopic control, right lateral fluoroscopic control showing the optimal placement of the guide wire
Tibial pin guide

Under arthroscopic control, the tibial guide is introduced through the anteromedial portal. The angle of tibial aimer is set to $40^{\circ}$ to secure the proper slope of the tunnel. A tibial tunnel at a constant angle without consideration to the patellar tendon length is carried out because the graft-tunnel mismatch is a non-issue in the two-incision technique. The landmark for ACL guide is a distance of $7 \mathrm{~mm}$ anterior to the PCL and on an average $7 \mathrm{~mm}$ lateral to the medial condyle. A longitudinal skin incision $3 \mathrm{~cm}$ inferior to the articular margin above the pes anserinus tendon and at least $1 \mathrm{~cm}$ medial to the tibial tubercle is carried out and a guide pin is drilled with the flexed knee at $90^{\circ}$. After the guide pin is placed, the drill guide is removed and the knee is passively extended to exclude an impingement on the anterior intercondylar notch region.

\section{Femoral tunnel}

Once a check is performed, femoral tunnel is at first established with a 11 or $10 \mathrm{~mm}$ cannulated reamer depending on the bone block size. A curet is used to cup the tip of the guidewire to avoid inadvertent advancement of the guidewire, causing damage to the articular surface or other intra-articular structures. The most anterior edge of the performed tunnel is located at the level of the isometric point. The reaming debris is evacuated with a synovial shaver to minimize the fat pad inflammatory response. The anterior edge of the tunnel, at its entrance into the joint, is chamfered with a curet to prevent abrasion of the graft as it exits through the femoral tunnel (Fig. 3).

\section{Tibial tunnel}

A 10 or $9 \mathrm{~mm}$ tibial tunnel depending on graft size is established with a cannulated reamer placed over the tibial guide pin. The tibial tunnel is usually 1 or $2 \mathrm{~mm}$ inferior in size with respect to the femoral tunnel. Once the tibial tunnel is performed, a motorized $4.5 \mathrm{~mm}$ full radius resector is introduced in a retrograde via the tibial tunnel to debride the intra-articular entrance. A plug can be placed in the tunnel and the shaver introduced through the anteromedial portal to further remove loose bone and cartilage around the tunnel entrance. Care is taken to ensure that residual tissue that may impinge on the graft does not remain at the anterior edge and the surgeon should smooth out the posterior and posterolateral intra-articular edge of the tibial tunnel. 


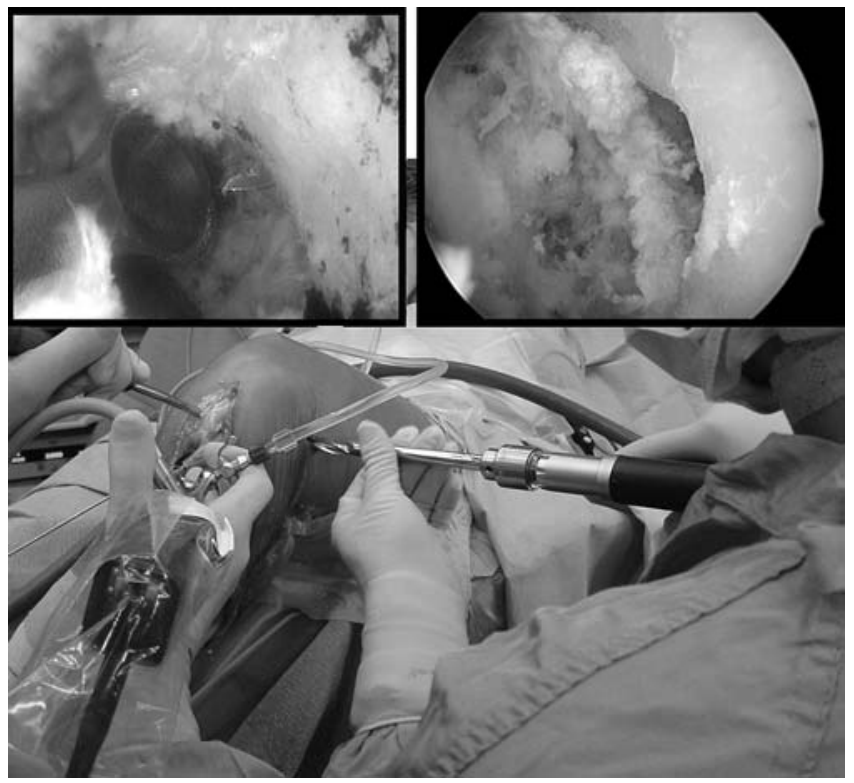

Fig. 3 Drilling of femoral tunnel from lateral incision. Left arthroscopic view of a curette above the tip of pin, right position of tunnel at footprint at the 9:30 position

\section{Graft passage, tensioning and fixation}

The BPTB autograft is passed antegrade from the femoral to tibial side with a pull-through technique under direct arthroscopic vision. A suture loop is introduced into the joint through the tibial tunnel using an eyed pin and is then pulled into the femoral tunnel under arthroscopic view and grasped by the assistant. The other side of the suture loop goes out of tibial tunnel. The assistant passes the pull sutures of the patella bone plug in the suture loop, so the surgeon pulling on the loop suture out of tibial tunnel allows the patella plug sutures to go out of the tibial tunnel (Fig. 4). At this point, verified with a clamp the direction of the femoral tunnel, the assistant grasps with a clamp the patella bone plug that is guided through the femoral tunnel. When the bone plug arrives at the intra-articular entrance of the femoral tunnel, the clamp is removed and the surgeon pulls on the traction suture to allow the passage of the graft. A probe can sometimes be necessary to assist the graft passage. The tibial block is inserted in the femoral tunnel, with the cortex face posteriorly. The surgeon performs a gentle traction distally while the assistant lightly hammers using an impactor inserted on the bone plug until the press-fit graft block in the tunnel, and the marked tendo-osseus junction is viewed arthroscopically to arrive at the intra-articular entrance of the femoral tunnel (Fig. 5). Once the graft is seated in the femoral tunnel, its position is well assessed with the arthroscope. A maximal manual tension is applied to the sutures of the patellar plug and the knee is cycled

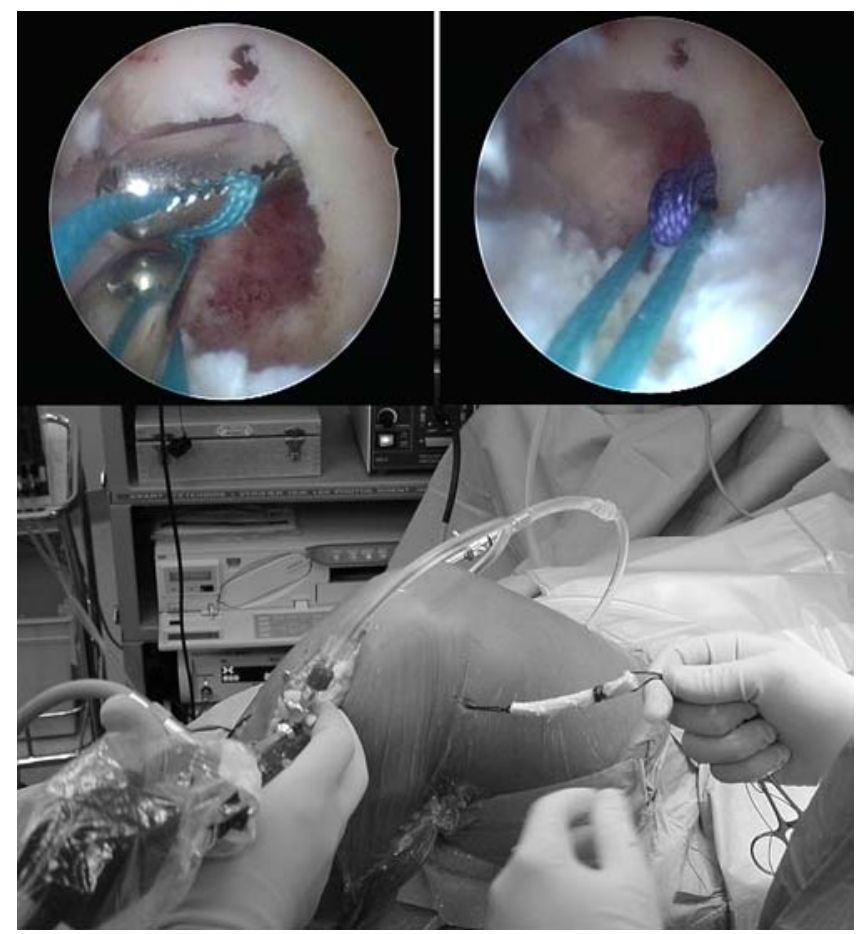

Fig. 4 Passage of BPTB graft in antegrade fashion. At the top the arthroscopic control of the sutures passage. A suture retriever is inserted trough the femoral tunnel and grasps the loop suture from the eyed pin. Loop suture is then passed out the lateral femoral cortex allowing the passage of graft

through full flexion extension several times for graft pretensioning and settling. The knee is placed in approximately $30^{\circ}$ of flexion, and tibial fixation is achieved with primary aperture-opening fixation using a $9 \mathrm{~mm}$ bioabsorbable interference screw (Phusis) placed anteriorly to the graft (Fig. 6). The scope inserted through the lateral portal controls the position of the screw at the articular margin of the tibial tunnel. After this, the scope is inserted retrograde in the tibial tunnel to control the proper position of the screw and verify that during passive motion of the knee there is an absence of graft motion. At last intraoperative stability is assessed with Lachman testing. Surgical incision is closed on an intra-articular suction drainage.

\section{Discussion}

The knowledge of the anatomy of the ACL and in particular its anatomical attachment site on the lateral femoral condyle is mandatory for successful ACL surgery $[3,11]$. The ACL femoral attachment is semicircular $(18 \times 10 \mathrm{~mm})$ with a straight anterior border and a convex posterior border as it originated from the posteromedial wall of the lateral femoral condyle. It lies behind 


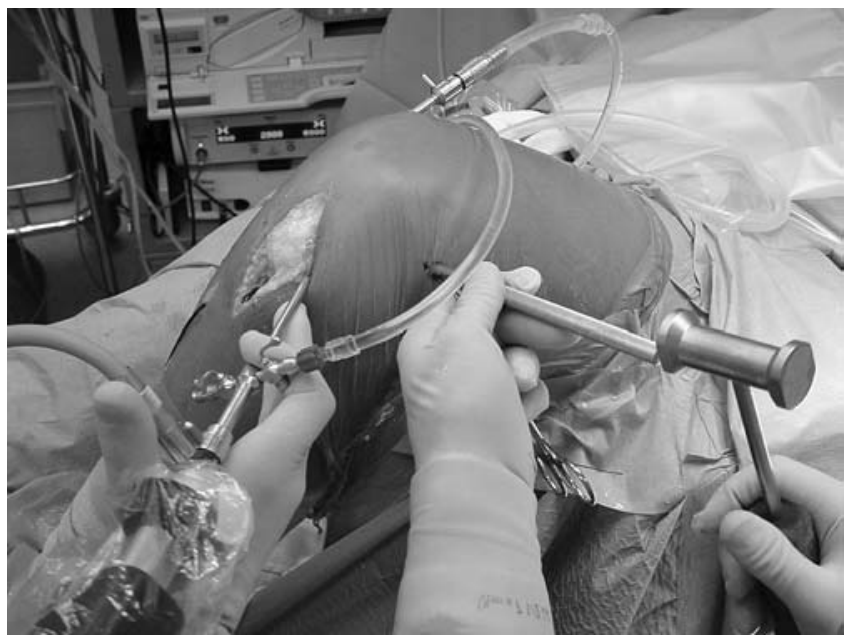

Fig. 5 The assistant hammering light using an impactor on the tibial bone plug while the surgeon performs traction distally on the sutures

and below the roof of the intercondylar notch and behind the inferior extension of the posterior femoral cortex, at the transition between the bony lateral notch wall and the cartilage of the femoral condyle $[2,16]$. The major part of current endoscopic ACL reconstruction techniques place the graft along the roof of the intercondylar notch, anterior and superior to the native ACL insertion, exposing some of fibres of the non-anatomically placed graft to higher strain rates and risk of failure. The ACL presents a collection of individual fibres that are a simplistic grouping in two distinct bands, anteromedial (AM) and posterolateral (PL) bands [16].

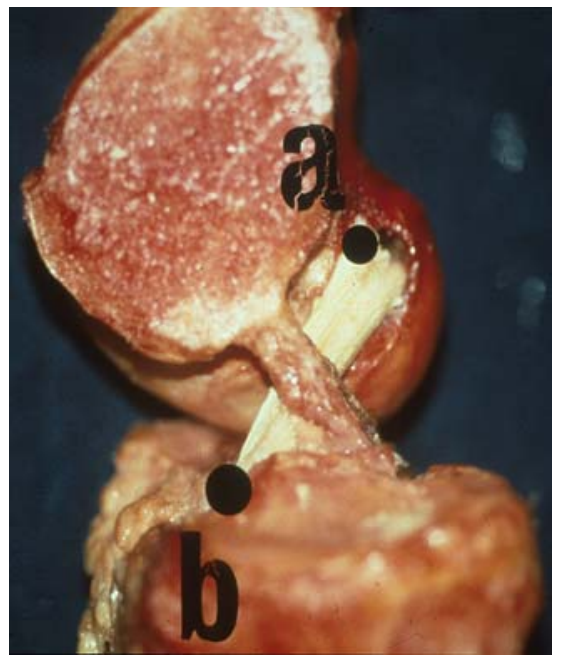

Fig. 6 Sagittal cross-section through the cadaveric knee shows the near anatomical position of graft $(a$ and $b)$ after simulate ACL reconstruction with the two-incision technique
The AM band is located in the anterior part of ACL and is inserted just behind and at the top of the intercondylar notch roof. This point corresponding to the most isometric point [12, 19, 23], consequently, the most anterior fibres of AM band are the most isometric [13, 18, 19, 29]. The PL band of the ACL represents the bulk of ligament and its fibres are the most inferior and posterior. It was well demonstrated by Hefzy et al. [19] that altering the femoral attachment of the ACL had a much larger effect than tibial attachment on the length change of the fibres. In fact, the centre of rotation is closer to the femoral attachment than the tibial attachment side, so there is little room for error when placing the femoral tunnel. Clinical results correlate positively with femoral tunnels placed at least $60 \%$ posterior to the anterior origin of Blumensaat line [21].

A cadaveric study confirmed that the anatomical insertion of ACL on the femur is found to lie very low in the notch [3]. The major part of these fibres lies posteriorly to the isometric point on the medial wall of the femoral condyle [17]. These fibres located behind the most isometric point are lax during flexion and tight in extension. The behaviour of these fibres was defined 'favourable non-isometry' [8]. A progressive recruitment of the fibres from front (the most isometric) to backward is the product during knee extension. The 'favourable non-isometry' is very interesting because increased knee loading often occurs at flexion angles of less than $60^{\circ}$, and to reproduce it, an anatomical placement of the graft should be performed during surgery.

In the past, the most common femoral tunnel error was to place the femoral tunnel too anteriorly resulting in a graft lax in extension, tight in flexion. The major part of the current endoscopic ACL reconstruction place the graft high in the most posterior aspect of the intercondylar roof notch, anterior and superior to the native ACL insertion. This position results in $10 \mathrm{~mm}$ of laxity from extension to flexion, and therefore, causes the graft to tighten excessively in knee extension [1]. Studies evaluating isometric placement of graft have suggested that the 12 o'clock position with a $2 \mathrm{~mm}$ of posterior wall was the most isometric [9]. In reality this position results in a vertically oriented non-anatomic graft [7]. In such a situation the anterior tibial translation is partially controlled, but the rotational component of the instability remains uncontrolled, resulting in a persistent pivot shift [14]. Hefzy et al. [19] noted a larger isometric $(2 \mathrm{~mm})$ zone proximally, so most authors are recommending an entry point high in the notch, at the 11 o'clock position for a right knee with $1-2 \mathrm{~mm}$ posterior cortical shell, and often this requires the use of a more inferior medial portal. Nevertheless, the ACL isometry is a theoretical condition, and has not stood up to detailed testing and investigation [9, 13, 24].

Most fibres of the ACL are actually quite non-isometric with only the most anterior fibres being near 
isometric. We think that anatomical placement of graft should be preferred, so the goal of femoral tunnel placement should be to place the graft in a position that most accurately reproduces an anatomic behaviour of the ACL.

A cadaveric study made by Arnold et al. [3] showed as the ACL attachment site to spread between 11 and 9-8 o'clock position; the centre lies lower than at 11 o'clock position, so to place the graft at the original anatomical insertion site a tunnel at 10 o'clock or lower should be placed. In this study it was found that transtibial femoral tunnel drilling does not reach the centre of the anatomical ACL footprint. It was concluded that femoral aiming devices for use through the tibial tunnel aim for an isometric placement, and they do not aim for an anatomic position of the graft. To reach a better femoral tunnel placement the medial portal instead of the transtibial portal with a knee flexed at $130^{\circ}$ has been proposed [28]. Simmons et al. recently [27], have showed that placing the femoral tunnel in the coronal plane at $60^{\circ}$ lowers graft tension in flexion minimizing PCL impingement of graft. To obtain this positioning with the transtibial technique, a special tibial guide should be used and drilling through the superficial fibres of the medial collateral ligament should be performed [27]. The femoral tunnel placement is very important because it not only determines graft tension [20] but also affects the ligamentization process of the graft healing. A recent study on a cadaveric biomechanical model has shown that reconstructing the femoral tunnel at the oblique anatomic origin of the native ACL, oriented $60^{\circ}$ from vertical, more closely restored anatomic rotational stability of the knee than did the standard tunnel reconstruction oriented at $30^{\circ}$ from vertical [25]. This corresponding to ACL reconstruction with a 'favourable isometry' and to place the graft in this area, a femoral tunnel between 9 and 10 o'clock position on the right lateral femoral condyle should be drilled, with the knee at $90^{\circ}$ of flexion. The posterior placement of graft and the horizontal femoral tunnel result in an anatomical placement, restoring more natural knee kinematics [2, 22]. The classsic two-incision technique, using a rearentry drill guide [15], our two-incision technique, or the Clancy anatomic endoscopic technique using flexible reamers and use of different not commonly arthroscopic portals [7] seems to allow a predictable, nearanatomic placement of femoral tunnel.

\section{References}

1. Acker JH, Drez D (1989) Analysis of isometric placement of grafts in ACL reconstruction procedures. Am J Knee Surg 2:65-70

2. Amis AA, Jacob RP (1998) Anterior cruciate ligament graft positioning, tensioning and twisting. Knee Surg Sports Traumatol Arthrosc 6(Suppl 1):S2-S12

3. Arnold MP, Kooloos J, van Kampen A (2001) Single-incision technique misses the anatomical femoral anterior cruciate ligament insertion: a cadaver study. Knee Surg Sports Traumatol Arthrosc 9(4):194-199

4. Bach BR Jr (1989) Arthroscopy assisted patellar tendon substitution for anterior cruciate ligament reconstruction. Am J Knee Surg 2:3-20

5. Bernard M, Hertel P, Hornung H, Cierpinski T (1997) Femoral insertion of the ACL. Radiographic quadrant method. Am J Knee Surg 10(1):14-21

6. Buss DD, Warren RF, Wickiewicz TL, Galinat BJ, Panariello R (1993) Arthroscopically assisted reconstruction of the anterior cruciate ligament with use of autogenous patellar-ligament grafts. Results after twenty-four to forty-two months. J Bone Joint Surg Am 75(9):1346-1355
7. Cain EL Jr, Clancy WG Jr (2002) Anatomic endoscopic anterior cruciate ligament reconstruction with patella tendon autograft. Orthop Clin North Am 33(4):717-725

8. Chambat P, Selva O (1999) Reconstruction du ligament croisè anterieure par autogreffe au tendon rotulien. Forage du tunnel fémoral de dehors en dedans. In: Sociéte Francaise d'Arthroscopie (eds) Elsevier, Paris, pp 144

9. Cooper DE, Urrea L, Small J (1998) Factors affecting isometry of endoscopic anterior cruciate ligament reconstruction: the effect of guide offset and rotation. Arthroscopy 14(2):164-170

10. Denti M, Bigoni M, Randelli P, et al (1998) Graft-tunnel mismatch in endoscopic anterior cruciate ligament reconstruction. Intraoperative and cadaver measurement of the intraarticular graft length and the length of the patellar tendon. Knee Surg Sports Traumatol Arthrosc 6(3):165-168

11. Fanelli GC, Desai BM, Cummings PD, et al (1994) Divergent alignment of the femoral interference screw in single incision endoscopic reconstruction of the anterior cruciate ligament. Contemp Orthop 28:21-25
12. Friederich NF, O'Brien WR (1990) Functional anatomy of the cruciate ligaments. In: Jacobs RP, Staubli HU (eds) The knee and the cruciate ligaments. Springer, Berlin Heidelberg New York

13. Furia JP, Lintner DM, Saiz P, Kohl HW, Noble P (1997) Isometry measurements in the knee with the anterior cruciate ligament intact, sectioned, and reconstructed. Am J Sports Med 25(3):346-352

14. Getelman MH, Friedman MJ (1999) Revision anterior cruciate ligament reconstruction surgery. J Am Acad Orthop Surg 7(3):189-198

15. Gill TJ, Steadman JR (2002) Anterior cruciate ligament reconstruction the two-incision technique. Orthop Clin N Am 33:727-735

16. Girgis FG, Marshall JL, Monajem A (1975) The cruciate ligaments of the knee joint. Anatomical, functional and experimental analysis. Clin Orthop 106:216-231

17. Giron F, Buzzi R, Aglietti P (1999) Femoral tunnel position in anterior cruciate ligament reconstruction using three techniques. A cadaver study. Arthroscopy 15(7):750-756 
18. Good L, Gillquist J (1999) The value of intraoperative isometry measurements in anterior cruciate ligament reconstruction: an in vivo correlation between substitute tension and length change. $\mathbf{J}$ Arthrosc Relat Surg 9:525-532

19. Hefzy MS, Grood ES, Noyes FR (1989) Factors affecting the region of most isometric femoral attachments. Part II: The anterior cruciate ligament. Am J Sports Med 17(2):208-216

20. Howell SM, Deutsch ML (1999) Comparison of endoscopic and two-incision techniques for reconstructing a torn anterior cruciate ligament using hamstring tendons. Arthroscopy 15(6):594606

21. Khalfayan EE, Sharkey PF, Alexander AH, et al. (1996) The relationship between tunnel placement and clinical results after anterior cruciate reconstruction. Am J Sports Med 24:335-341
22. Loh JC, Fukuda Y, Tsuda E, Steadman RJ, Fu FH, Woo SL (2003) Knee stability and graft function following anterior cruciate ligament reconstruction: comparison between 11 o'clock and 10 o'clock femoral tunnel placement. Arthroscopy 19(3):297-304

23. Noyes FR, Butler DL, Grood ES, Zernicke RF, Hefzy MS (1984) Biomechanical analysis of human ligament grafts used in knee-ligament repairs and reconstructions. J Bone Joint Surg Am 66(3):344-352

24. Sapega AA, Moyer RA, Schneck C, et al. (1990) Testing for isometry during reconstruction of the anterior cruciate ligament: anatomical and biomechanical considerations. J Bone Joint Surg Am 72:259-267

25. Scopp JM, Jasper LE, Belkoff SM, Moorman CT III (2004) The effect of oblique femoral tunnel placement on rotational constraint of the knee reconstructed using patellar tendon autografts. Arthroscopy 20(3):294-299

26. Sgaglione NA, Schwartz RE (1997) Arthroscopically assisted reconstruction of the anterior cruciate ligament: initial clinical experience and minimal 2-year follow-up comparing endoscopic transtibial and two-incision techniques. Arthroscopy 13(2):156-165
27. Simmons R, Howell SM, Hull ML (2003) Effect of the angle of the femoral and tibial tunnels in the coronal plane and incremental excision of the posterior cruciate ligament on tension of an anterior cruciate ligament graft: an in vitro study. J Bone Joint Surg Am 85(6):1018-1022

28. Webb JM, Corry IS, Clingeleffer AJ, Pinczewski LA (1998) Endoscopic reconstruction for isolated anterior cruciate ligament rupture. J Bone Joint Surg Br 80(2):288-294

29. Zavras TD, Race A, Bull AM, Amis AA. (2001) A comparative study of 'isometric' points for anterior cruciate ligament graft attachment. Knee Surg Sports Traumatol Arthrosc 9(1):28-33 